\title{
Morbus Bechterew
}

Die Bedeutung der genetischen Prädisposition
für die Beurteilung der Diensttauglichkeit
an einem Fallbeispiel der Spondylitis ankylosans

F. Frey ${ }^{a}$, H. A. Schwarz ${ }^{b}$, S. van der Linden ${ }^{c}$

a Generalstab, Untergruppe Sanität, Sektion Militärärztlicher Dienst, Kreisarzt III

b Chefarzt Klinik für Rheumatologie und Rehabilitation, Bethesda-Spital Basel

c Professor of Rheumatology, University Hospital Maastricht, Netherlands

\section{Résumé}

Un conscrit en bonne santé, mais avec un facteur HLA-B27 positif, des parents du premier degré souffrant manifestement d'une maladie de Bechterew, a été jugé inapte au service. Le présent cas met en évidence le problème de l'évaluation des prédispositions génétiques pour poser un diagnostic et apprécier les suites possibles de l'affection.

En 1986, le tribunal fédéral des assurances a reconnu une responsabilité à vie de l'assurance militaire pour le père qui avait souffert d'une première poussée de spondylarthrite ankylosante dans le cadre du service militaire. L'expertise sur laquelle se fonde le jugement fait état d'une causalité se situant entre des facteurs environnementaux (activité physiquement éprouvante, froid, humidité) et la poussée de la maladie.

L'évaluation de la prédisposition génétique n'est généralement pas une condition préalable pour l'appréciation de l'aptitude au service. C'est l'unique cas pour lequel, en présence d'une prédisposition génétique, spécialement et avec de surcroît une anamnèse familiale lourde, une inaptitude au service a été acceptée.

Dans le domaine civil (renseignements obtenus auprès de l'administration fédérale), il n'est pas tenu compte de la prédisposition génétique pour un emploi, également dans des professions exposées.

La question de l'inaptitude au service, posée uniquement sur la base de facteurs génétiques et d'une anamnèse familiale lourde doit être écartée et à nouveau être appréciée. La reconnaissance d'une inaptitude au service chez des conscrits sains reposant uniquement sur une hypothèque génétique familiale ne semble plus être justifiée.

\section{Einleitung} relle Voraussetzung. Beim Vorliegen der genetischen Prädisposition bei Morbus Bechterew wurde bisher im Einzelfall, speziell bei zusätzlicher familiärer Belastung, auch bei bisher gesunden Probanden eine Dienstuntauglichkeit angenommen.

Im zivilen Bereich (abgeklärt für die Bundesverwaltung) wird die genetische Prädisposition für die Anstellung auch in exponierten Berufen nicht berücksichtigt.

Die Frage der Dienstuntauglichkeit allein aufgrund genetischer Faktoren und familiärer Belastung soll aufgerollt und neu beurteilt werden. Die Anerkennung einer Dienstuntauglichkeit bei gesunden Stellungspflichtigen rein aufgrund genetischer und familiärer Belastung scheint nicht mehr gerechtfertigt.
Kontaktadresse:

Dr. med. F. Frey

GST, UG San

Sektion Militärärztlicher Dienst

CH-3003 Bern

Tel. 0313242731
Von einer genetischen Prädisposition wird bei Krankheiten gesprochen, deren Vorkommen mit einem bestimmten genetischen Merkmal assoziiert ist.

Am Beispiel der Spondylitis ankylosans wird im folgenden Artikel die Bewertung von genetischen Prädispositionen zur Prävention der Auslösung von Krankheiten diskutiert.

Welche Konsequenzen ergeben sich aus der Beantwortung dieser Frage für die Beurteilung der Diensttauglichkeit und für die Arbeitsfähigkeit in physisch belastenden Berufen der Bundesverwaltung? Muss in Zukunft zum Ausschluss von genetisch bedingten Risiken eine Genanalyse bei der Aushebung für den Militärdienst oder vor einer Anstellung bei der Bundesverwaltung gefordert werden?

\section{Fallbeschreibung}

1999 wurde ein gesunder 19jähriger Stellungspflichtiger bei der Aushebung dienstuntauglich erklärt. Anamnestisch und aktuell waren keine Rücken- oder 
Gelenkschmerzen eruierbar. Grund für die Dienstuntauglichkeit waren die familiäre Belastung mit Morbus Bechterew (Vater und Onkel) und der positive Nachweis des Histokompatibiliätsantigens HLA-B27 beim Stellungspflichtigen.

Dieser hatte vor seiner Aushebung Unterlagen über den Verlauf der Krankheit bei seinem Vater vorgelegt: Sein Vater absolvierte vor 25 Jahren eine Rekrutenschule, wobei Rückenschmerzen auftraten, die sich im weiteren Verlauf als erster Schub einer Spondylitis ankylosans erwiesen. Er wurde daraufhin dienstuntauglich erklärt. Die Militärversicherung übernahm die Kosten für zwei Jahre, lehnte aber eine weitere Bundeshaftung mit der Begründung ab, dass der Militärdienst den Morbus Bechterew nicht verursachen, wohl aber - gleich den Schubkrankheiten vorübergehend verschlimmern könne. Mit einer Verwaltungsgerichtsbeschwerde wurde das Eidgenössische Versicherungsgericht angerufen, das mit Entscheid von 1986 die lebenslange Haftung der Militärversicherung in diesem Fall als gegeben ansah.

Gestützt wurde dieses Urteil auf ein 1980 erstelltes Gutachten einer Universitätsklinik. Der Gutachter hält es «nicht nur für denkbar, sondern höchst wahrscheinlich, dass für das Manifestwerden einer klinisch stummen Ileosakralarthritis besondere äussere Einwirkungen (z.B. überdurchschnittliche körperliche Belastung oder ungünstige Witterungsverhältnisse wie sie im Militärdienst vorkommen können) die klinisch stumme Entzündung zu einem subjektiven Leiden, d.h. zu einer Krankheit umzuwandeln vermögen".

In einem vom Stellungspflichtigen ebenfalls eingereichten aktuellen Zeugnis seines Hausarztes wurde eine Berücksichtigung der genetischen Prädisposition im Sinne einer "eingeschränkten" Diensttauglichkeit beantragt. Wegen des Fehlens von klinischen Symptomen wurde auf Beckenröntgenaufnahmen verzichtet.

\section{Klinik der Spondylitis ankylosans}

Die Spondylitis ankylosans (Morbus Bechterew) gehört zu den seronegativen Spondylarthropathien, die mit gehäuftem Auftreten von HLA-B27 assoziiert sind. Eine auslösende Rolle ist für infektiöse Faktoren (urogenitale und gastrointestinale Infekte) empirisch sehr wahrscheinlich [1, 7] und wird durch Tiermodelle (vgl. unter "Ätiopathogenese der Spondylitis ankylosans") gestützt. Die Prävalenz beträgt in der kaukasischen Bevölkerungsgruppe mindestens 0,5\% [2, 3], wobei die Männer 3- bis 5mal häufiger betroffen sind als die Frauen [2, 3]. Es gibt eine juvenile Form mit Beginn vor dem 16., meist um das 12. bis 13. Lebensjahr $[4,5]$.

\section{Frühzeichen}

Leitsymptom der Frühphase sind nächtliche Glutaealund Kreuzschmerzen, die typischerweise in Ruhe exazerbieren und auf Bewegung hin deutlich nachlassen (ca. 75\%) [4]. Meist sind sie symmetrisch oder werden es innert weniger Monate. Frühzeitig können sich auch Insertionstendinitiden (z.B. am Kalzaneus) entwickeln. Eine Uveitis als Frühsymptom kommt in 3\% der Fälle vor [3, 5]. Die juvenile Form beginnt praktisch immer mit einer peripheren Arthritis und nicht mit der Rückensymptomatik [4, 5].

\section{Vollbild}

Die oft, aber keineswegs immer fortschreitenden, aufsteigenden Veränderungen der Wirbelsäule (mit charakteristischen radiologischen Zeichen: Syndesmophyten, Kastenwirbel, Shiny Corners der Wirbelkörper) gehen mit zunehmenden, anfangs oft schmerzhaften, thorakalen und zervikalen Funktionseinschränkungen einher. Arthritiden treten bevorzugt stammnahe oder in den unteren Extremitäten, meist asymmetrisch auf (in ca. 1/3 der Fälle). Im Spätstadium kann sich eine zunehmende Deformierung mit fixierter Fehlhaltung der Wirbelsäule entwickeln.

\section{Viszerale Beteiligung}

Häufigste extraartikuläre Manifestation sind Enthesopathien und mit 25\% die Uveitis [4]. Selten sind Aorteninsuffizienz, Herzrhythmusstörungen, apikal betonte Lungenfibrose, Glomerulonephritis und neurologische Störungen.

\section{Prognose}

Der Verlauf der Krankheit ist sehr variabel. Charakteristisch sind spontane Remissionen und Exarzerbationen, die sich über Jahre und Jahrzehnte erstrecken können. Die meisten Patienten mit ankylosierender Spondylitis führen ein durchaus normales Leben und bleiben voll arbeitsfähig [5-7]. Die Lebenserwartung scheint nur unter ungewöhnlichen Umständen (Komplikationen bei viszeraler Beteiligung; Therapiekomplikationen) verkürzt [5-7].

Die Diagnose- und Klassifikationskriterien eines Morbus Bechterew sind aus Tabelle 1 ersichtlich.

\section{Ätiopathogenese der Spondylitis ankylosans}

\section{Die Rolle von HLA-B27}

Die von Brewerton 1973 entdeckte Korrelation zwischen dem Histokompatibilitätsantigen HLA-B27 und der Spondylitis ankylosans sowie weiteren, unter dem Sammelbegriff der «seronegativen Spondylarthropathien" zusammengefassten Krankheiten konnte in den letzten Jahren weiter geklärt werden. So untermauern epidemiologische Untersuchungen den $\mathrm{Zu}-$ sammenhang zwischen der Prävalenz von HLA-B27 und dem Vorkommen von Spondylitis ankylosans: Beide sind praktisch nicht vorhanden bei Bantus und Buschmännern im südlichen Afrika und bei den australischen Aborigines, hingegen sehr häufig bei den Haida-Indianern Nordamerikas (ca. 50\% positiv) [2]. In Westeuropa kommt HLA-B27 in 6 bis 9\% [2, 3, 5] der Normalbevölkerung und bei rund 95\% der Patienten mit Spondylitis ankylosans vor $[2,5,8]$. 


\section{Tabelle 1}

Modifizierte New-York-Kriterien des Morbus Bechterew

(nach van der Linden und Mitarbeiter [3, 19]).

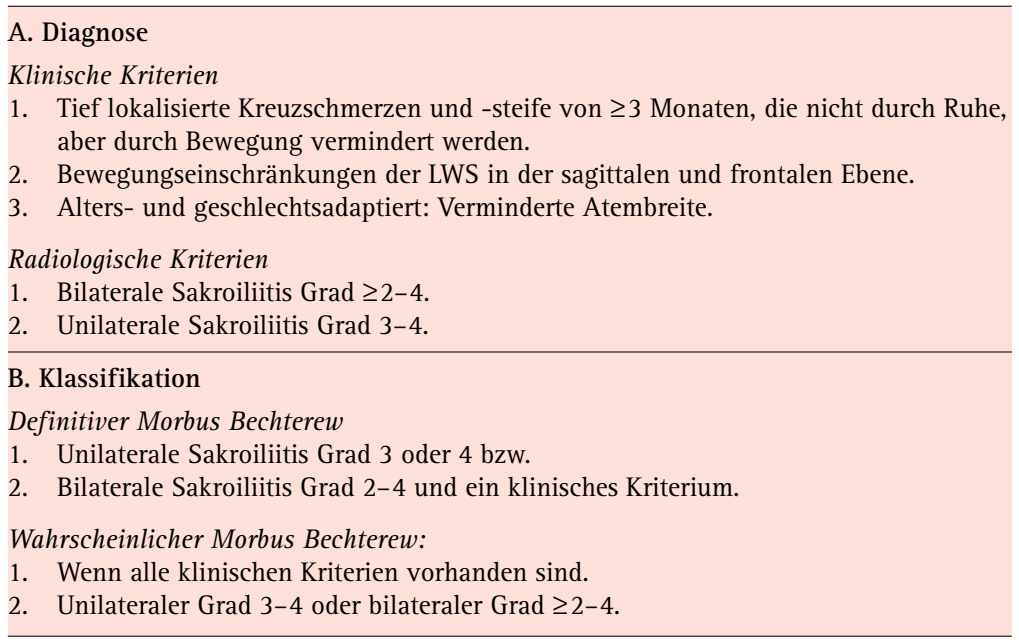

\section{Subtypen}

20 Subtypen von HLA-B27 sind charakterisiert worden, bezeichnet als $B^{*} 2701-B^{*} 2720$ [9]. Der bei uns weitaus häufigste Subtyp B*2705 (in Westeuropa bei 90 bis 96\% vorhanden [3]) ist stark assoziiert mit Spondylitis ankylosans.

Tiermodelle weisen ebenfalls darauf hin, dass HLA-B27 direkt in die Pathogenese der Spondylarthropathien involviert ist, allerdings unter obligater Mithilfe von Bakterien. Transgene HLA-B27-Mäuse entwickeln keine Arthritis, solange sie in keimfreier Umgebung gehalten werden [3, 10]. Nach Wechsel in eine normale Umgebung entwickelt sich die Arthritis jedoch rasch. Die Arthritisanfälligkeit korreliert bei Ratten zudem mit dem Grad der HLA-B27-Genexpression [11]. Diese und andere Tiermodelle und Experimente zeigen, dass HLA-B27 selbst und nicht ein nahe verwandtes Gen für die Arthritis verantwortlich ist.

\section{Nicht HLA-B27-Gene}

Die Studien beim Menschen und die Resultate von transgenen Tiermodellen und Experimenten zeigen, dass neben dem direkt verantwortlichen HLA-B27Gen andere Gene eine wesentliche Rolle spielen. So erhöht HLA-B60 allein oder mit einem Link-Gen zusammen die Anfälligkeit für Spondylitis ankylosans, während HLA-DR-Gene das Erkrankungsrisiko nur unwesentlich erhöhen [12].

Polymorphismus von Genen, welche z.B. den Tumor-Nekrosis-Faktor, Proteasomen u.a. kodieren, kann ebenfalls die Krankheitsanfälligkeit oder Ausprägung beeinflussen [13].

\section{Diskussion}

Für die Beurteilung der Diensttauglichkeit bei Morbus Bechterew gilt gemäss den einschlägigen Richtlinien (Nosologia Militaris):
Stellungspflichtige/Rekruten mit einem gesicherten Morbus Bechterew sind zwingend dienstuntauglich. Bei Ausexerzierten erfolgt die Beurteilung gemäss fachärztlichem Zeugnis.

Beim beschriebenen Fall des Stellungspflichtigen liegt neben der genetischen Prädisposition eine positive Familieanamnese vor. Aufgrund dieser Fakten entschied die medizinische Untersuchungskommission bei der Aushebung auf dienstuntauglich, obwohl die Bedingungen der Richtlinien eines "gesicherten" Morbus Bechterew (vgl. Tab. 1) keineswegs erfüllt waren.

Dieser Entscheid wurde im Spannungsfeld der Einhaltung des Grundsatzes der allgemeinen Wehrpflicht (dieser Stellungspflichtige ist ja [noch] gesund!) und der individuellen Risikoabschätzung von möglichen Erkrankungen gefällt. Es stellt sich aber die Frage, ob dieser Entscheid auch begründbar ist und was er bei genereller Anwendung implizieren würde.

Die Prävalenz der Krankheit würde bei der Kohortengrösse von jährlich 33000 Stellungspflichtigen eine Zahl von mindestens 160 Patienten erwarten lassen. Effektiv werden durchschnittlich etwa 50 Stellungspflichtige bereits an der Aushebung wegen eines klinisch manifesten Morbus Bechterew von der Dienstpflicht befreit. Das lässt sich durch die Altersverteilung bei der Diagnosenstellung erklären.

Würde für die Beurteilung der Diensttauglichkeit bei der Aushebung ein HLA-B27-Test gefordert und wäre dieser bei Positivität ein Kriterium gegen die Diensttauglichkeit, so müssten jährlich $8 \%$, also etwa 2600 Stellungspflichtige deswegen dienstuntauglich erklärt werden. Davon würden nur etwa 2\% (130 Stellungspflichtige) wahrscheinlich später an einer Spondylitis ankylosans erkranken [7, 10].

Würde als Zusatzkriterium ein manifester Verlauf der Krankheit im engsten Familienkreis (Geschwister, Eltern) definiert, könnte die Zahl der Untauglichen wieder etwas eingeschränkt werden. Das HLA-B27 wird bei 50\% der erstgradig Verwandten von HLAB27-positiven Patienten gefunden [5]. Diese Verwandten haben ein Risiko von 10 bis 20\%, an einer Spondylitis ankylosans zu erkranken [3, 7, 8]. Das relative Risiko erhöht sich noch einmal um Faktor 3, wenn zusätzlich eine HLA-B60-Positivität vorliegt [3, 10]. Mit Einbezug des Kriteriums der HLA-B60Positivität liesse sich die Zahl der Untauglichen weiter einschränken.

Sind genetische Analysen grundsätzlich ein brauchbares Zusatzkriterium für die Beurteilung der Diensttauglichkeit? Diese Frage soll am Beispiel des Morbus Bechterew diskutiert werden.

- Die ätiopathogenetische Rolle des HLA-B27 bei der Spondylitis ankylosans ist unbestritten $(2,4$, 5, 7).

- Beim Morbus Bechterew sind gut dokumentierte Fälle mit negativem HLA-B27-Antigen bekannt $(5,10,14)$.

- In der weissen Normalbevölkerung kommt diese Genkonstellation in 8\% ebenfalls vor. Das Risiko der HLA-B27-Positiven an Spondylitis ankylosans zu erkranken, liegt lediglich bei $2 \%(7,10)$. 
Schlussfolgerung: Die generelle Durchführung eines genetischen Screeningtests auf HLA-B27 für die Beurteilung der Diensttauglichkeit ist nicht sinnvoll.

Auch als genereller diagnostischer Screeningtest für die Spondylitis ankylosans wird die HLA-B27Bestimmung in der Literatur nicht empfohlen [4]. Ein genetischer Screeningtest ist auch für gesunde, erstgradig Verwandte von Bechterew-Patienten nicht indiziert

Als Kriterium für die Durchführung eines HLAB27-Tests ist die Klinik (Anamnese, Symptome) massgebend. Im übrigen gilt, dass ein nicht nachweisbares HLA-B27 grösseren diagnostischen und differentialdiagnostischen Wert hat als ein nachgewiesenes [3].

Im Gegensatz zu anderen Krankheiten mit familiärer Prädisposition (z.B. Diabetes mellitus, multiple Sklerose etc.), wird bei der Spondylitis ankylosans die Eigenheit diskutiert, dass klinisch bisher stumme Sakroiliitiden durch Umgebungsfaktoren $[8,10]$ aktiviert werden können. Eine französische Studie aus dem Jahre 1990 [15] zeigt, dass körperlich belastende Arbeiten, Kälte- oder Feuchtigkeitsexposition zu häufigeren und schwereren Exazerbationen des Morbus Bechterew führen. Eine Kausalität zwischen diesen Faktoren und dem Ausbruch der Krankheit konnte jedoch nicht belegt werden.

Ausgelöst wird die Spondylitis ankylosans mit grosser Wahrscheinlichkeit durch ubiquitäre Bakterien, die im Militärdienst nicht signifikant häufiger auftauchen als im Zivilleben. Bei Risikogruppen (erstgradig erkrankten Verwandten, HLA-B27- und eventuell sogar HLA-B60-Positivität oder entzündlichen Augenproblemen) muss überlegt werden, ob nicht auch ohne signifikante klinische Symptome im konventionellen Beckenröntgen Hinweise auf eine stumme Sakroiliitis gesucht werden müssen (vgl. Tab. 1). Dies hinsichtlich der Tatsache, dass bei $99 \%$ der Morbus-Bechterew-Patienten sich die ersten pathologischen Röntgenbefunde am Ileosakralgelenk entwickeln [3]. Bei positivem Resultat wäre dann wohl vorsichtigerweise von der Exposition mit den erwähnten, vermeidbaren Faktoren (Feuchtigkeit, Kälte etc.) abzusehen. Dies müsste bei der Truppen- und Funktionseinteilung der Angehörigen der Armee berücksichtigt werden; wenn dies nicht möglich ist, müsste als Ausnahmefall eine Dienstuntauglichkeit ausgesprochen werden.

Zusammenfassend kann gesagt werden, dass heute im Lichte der neueren Forschungsergebnisse der beschriebene Fall eines noch gesunden Stellungspflichtigen als diensttauglich beurteilt werden müsste.

Bleibt die Haftungsfrage nach erstmaligem Auftreten der Spondylitis ankylosans im Militärdienst zu diskutieren. Beim erstmaligen Auftreten einer Spondylitis ankylosans während des Militärdienstes haf- tet die Militärversicherung dauernd. Dies ist mit dem im Bundesgesetz über die Militärversicherung vom 19. Juni 1992 (Stand 1. Juli 1996) verankerten Kontemporalitätsprinzip zu erklären und bedeutet, dass die Militärversicherung für alle Gesundheitsschädigungen (also Krankheiten und Unfälle) und ihre Folgen, welche während einer Militärdienstleistung ihren Anfang genommen haben (bei denen also mit Sicherheit keine sogenannte Vordienstlichkeit nachgewiesen werden kann), Leistungen bis zur Heilung erbringen muss. Eine kausale Beziehung zwischen Gesundheitsschädigung und Militärdienst ist in einem solchen Falle nicht erforderlich.

Trotzdem ist nach aktuellen Aussagen der Militärversicherung [16] das Vorliegen einer HLA-B27Positivität per se kein Grund für eine Dienstuntauglichkeit. An der bisherigen Gewichtung der genetischen Prädispositionen soll aus dieser Sicht nichts geändert werden.

Im zivilen Bereich der Bundesbetriebe wird die Bewertung einer genetischen Prädisposition für die Berufstätigkeit gleich gehandhabt. Bei der Anstellung militärischer Berufsleute (MV-Vollversicherte) ist für die Untergruppe Sanität, als medizinisch verantwortliche Stelle, die genetische Prädisposition grundsätzlich kein Kriterium. Auch für den Ärztlichen Dienst der Bundesverwaltung [17] ist bei der medizinischen Eignungsabklärung von Swisscom-, PTT- und SBBAngestellten für körperlich belastende und/oder kälte- und feuchtigkeitsexponierte Arbeitsorte (wie Postangestellte im Aussendienst, Gleisarbeiter etc.) die genetische Prädisposition bei asymptomatischen Bewerbern kein Hinderungsgrund für deren Anstellung. Selbstverständlich wird bei Vorliegen dieser Konstellation der Stellenbewerber über die möglichen Risiken informiert.

\section{Schlussfolgerung}

Eine genetische Disposition ist per se kein Grund für eine Dienstuntauglichkeit und ebenfalls kein Grund für einen Vorbehalt bei einer Anstellung beim Bund. Diese am Beispiel des Morbus Bechterew gemachte Aussage kann aus heutiger Sicht generell auf alle Krankheiten mit genetischer Disposition ausgeweitet werden.

Interessant in diesem Zusammenhang ist eine Pressemeldung vom Februar dieses Jahres [18], wonach US-Präsident Clinton es ablehne, dass Stellenbewerber genetisch getestet werden. Clinton erliess deshalb ein Verbot an alle Bundesbehörden, solche Testergebnisse bei der Einstellung von Mitarbeitern $\mathrm{zu}$ berücksichtigen und forderte den Kongress auf, dies ebenfalls der Privatindustrie und den Versicherungsgesellschaften gesetzlich zu untersagen. 


\section{Literatur}

1 Wright V, Moll JMH. Seronegative Polyarthritis. Amsterdam: North Holland Publishing Co.; 1976.

2 Gram JT, Husby G. Ankylosing spondylitis: Prevalence and Demography. In: Klippel JH, Dieppe PA. Rheumatology. London, Philadelphia: Mosby, 1998. Section 6, chapter 15, page $1-6$.

3 Miehle W. Spondarthritiden: Spondylitis ankylosans. In: Miehle W, Fehr K, Schattenkirchner M, Tillmann K. Rheumatologie in Praxis und Klinik. Stuttgart, London: George Thieme Verlag; 2000. S. 629-75.

4 Khan MA. Ankylosing spondylitis: Clinical features. In: Klippel JH, Dieppe PA. Rheumatology. London, Philadelphia: Mosby, 1998. Section 6, chapter 16, page 1-16.

5 Kalden JR. Klinische Rheumatologie. Berlin, Heidelberg: Springer Verlag; 1998. S. 215-52.

6 Taurog JD, Lipsky PE. Spondylitis ankylosans, reaktive Arthritis und undifferenzierte Spondylarthropathie.

In: Harrison - Prinzipien der Inneren Medizin. Basel: Schwabe \& Co AG; 1995. S. 1940-3.

7 Khan MA, van der Linden S. Ankylosing spondylitis and other spondylarthropathies. Rheum Dis Clin North Am 1990; 16(3):551-79.

8 Ball GV. Ankylosing Spondylitis. In: Koopman WJ (ed.). Arthritis and Allied Conditions. A Textbook of Rheumatology. Philadelphia, London: Lippincott, Williams \& Wilkins; 1993. p. 1051-9.

9 Alvarez I, Lopez de Castro JA. HLA-B27 and immunogenetics of spondylarthopathies. Curr Opin Rheumatol 2000;12: 248-53.
10 Märki-Hermann E, Sucké B, Meyer zum Büschenfeld K-H. Neue Aspekte zur Pathogenese des Morbus Bechterew. Z Rheumatol 1996;55:4-18.

11 Taurog JD, Maika SD, Simmons WS, Breban M, Hammer RE. Susceptibility to inflammatory disease in HLA-B27 correlates with the level of B27 expression. J Immunol 1993;150: 4168-78.

12 Brown MA, Kennedy LG, Darke C, Gibson K, Pile KD, Shatford JL, et al. The effect of HLA-DR genes on susceptibility to and severity of ankylosing spondylitis. Arthritis Rheum 1998;41:460-5.

13 Fraile A, Nieto A, Vinasco J, Beraun Y, Martin J, Mataran L. Association of large molecular weight proteasome 7 gene polymorphism with ankylosing spondylitis. Arthritis Rheum 1998;41:560-2.

14 Gerber N, et al. HLA-B27-Negativität bei klinisch-klassischer Spondylitis ankylosans: Keine eigenständige nosologische Entität. Z Rheumatol 1977;36:224-9.

15 Guillemin F, Briançon S, Pourel J, Gaucher A. Long-term disability and prolonged sick leaves as outcome measurements in ankylosing spondylitis. Arthritis Rheum 1990;33(7): 1001-6.

16 Mündliche Mitteilungen von F. Gebel, Chefärztin, Bundesamt für Militärversicherung, Bern, 2000.

17 Mündliche Mitteilungen von P. A. Voumard, Chefarzt, Ärztlicher Dienst der SBB, der allgemeinen Bundesverwaltung, der PTT und der Swisscom, Bern, 2000.

18 Schweizerische Depeschenagentur, Bern, 9. Februar 2000.

19 van der Linden S, Valkenburg A, Cats A. Evaluation of diagnostic criteria for ankylosing spondylitis: A proposal for modification of the New York criteria. Arthritis Rheum 1984;27:361-8. 